Part of Journal of Research of the National Bureau of Standards, Volume 22, January 1939

\title{
AN IMPROVED RADIO METEOROGRAPH ON THE OLLAND PRINCIPLE
}

\author{
By L. F. Curtiss, A. V. Astin, L. L. Stockmann, and B. W. Brown
}

\section{ABSTRACT}

A description is given of the construction and tests of a radio telemeter of high precision built according to the Olland principle, in which all indicating arms rotate on a common axis. Combined with a 5-meter radio transmitter of push-pull type, this telemeter provides a radio meteorograph which has been tested under actual working conditions and found to be reliable and accurate in its indications. Since it gives relatively strong signals with a good stability in frequency, it is easy to operate and very little retuning during an observation is required. Special attention has been given to the thermal insulation of the compartment containing batteries and transmitter so that no failures due to drop in battery temperature have been observed even at altitudes above 60,000 feet. The cost of manufacture compares very favorably with that of other models recently developed in this country.

\section{CONTENTS}

\section{INTRODUCTION}

In September 1935 the authors described ${ }^{1}$ the results of their preliminary investigation into the possibilities of developing a practical system for observation of temperature, pressure, and relative humidity at high altitudes by means of radio-equipped sounding balloons. As there reported, the radio problems involved are very simple, and by using the Olland principle, which requires the transmitter to be in operation for a very small fraction of the observation period, relatively strong signals of 5-meters wave length can be obtained at all attainable altitudes and for distances of a hundred miles or more.

Attention has since been turned to the development of a radio telemeter which would be accurate in its indications and convenient to attach to a sounding balloon. In this period many models have been tested both in the laboratory and in the air. The chief problems encountered were those of: (1) Reliable keying contacts; (2) consistent indicating system for the sensitive elements; (3) adequate ventilation for the sensitive elements; (4) stable antenna position during flight; (5) maintenance of battery temperatures; and (6) dependable and uniform operation of the driving mechanism. The model here described has shown in repeated tests that these problems have been solved to the extent that they no longer interfere with reliable and accurate operation of the instrument.

${ }^{1}$ L. F. Curtiss and A. V. Astin, J. Aeron. Sci. 3, 35 (1935). 


\section{RADIO TELEMETER}

The preliminary experiments were made with clock-driven equipment. These trials served a very useful purpose in testing the range and stability of the radio signals, but it soon became evident that escapement errors introduced by the clock movement seriously limited the accuracy. Furthermore, even slight decreases in temperature frequently caused the clockwork to stop, especially if the pressure on the contact arms carried by the motor was sufficient always to give dependable signals. Tests were therefore made of small directcurrent motors, ${ }^{2}$ which convinced us that they would provide a more satisfactory driving mechanism. Slow changes in speed could be corrected for automatically ${ }^{3}$ in reading the record. Through cooperation of manufacturers small electric motors were made available. These weighed less than 50 grams and required about 5 milliamperes at 1.5 volts for their operation. Tests of their speed fluctuations revealed that the slow variations present could be kept to less than a few percent. In addition, these motors rarely stop in flight since they operate without lubricants and have about 100 times the power of a spring-driven clock of the same weight.

To protect the batteries and transmitter from excessive reductions in temperature, a thermally insulated inclosure is required. Likewise, the motor and electrical contacts for keying the transmitter should be protected from icing and other unfavorable conditions. We have found that a box with cellular walls made up of several layers of corrugated paper is best for this purpose. This inclosure is built up of several layers of corrugated paper glued together. The thermal insulation secured in this way is far superior to that provided by balsawood containers. This is shown by the following values which give the interior temperatures at approximately 5-minute intervals after surrounding the outside of the thermal inclosure with dry ice, $-78^{\circ} \mathrm{C}$. An inclosure with $1 / 4$-inch balsa-wood walls will drop to dry-ice temperature in about 12 minutes under the same conditions.

\begin{tabular}{|c|c|}
\hline Time & $\begin{array}{c}\text { Inside temper- } \\
\text { ature }\end{array}$ \\
\hline & $\circ C$ \\
$1: 17$ & 28 \\
$1: 22$ & 25 \\
$1: 30$ & 6 \\
$1: 35$ & 2 \\
$1: 40$ & -6 \\
$1: 45$ & -7 \\
$1: 55$ & -10 \\
$2: 00$ & -13 \\
\hline
\end{tabular}

It should be pointed out that Cellophane inclosures of the type described previously ${ }^{4}$ are in every way superior for maintaining the temperature of the battery inclosure at a high level in comparison with the external temperatures at the higher altitudes when the sun is above the horizon. For this reason Cellophane inclosures are still used by the authors ${ }^{5}$ for cosmic-ray balloon equipment, which is

\footnotetext{
2 L. F. Curtiss and A. V. Astin, Rev. Sci. Instr. 7, 358 (1936).

3 The authors are indebted to D. M. Little and A. H. Mears of the U. S. Weather Bureau for a simple device for this purpose.

${ }_{4}^{4}$ L. F. Curtiss and A. V. Astin, J. Aeron. Sci. 3. 35 (1935).

S L. F. Curtiss, A. V. Astin, et al., Phys. Rev. 53, 23 (1938).
} 


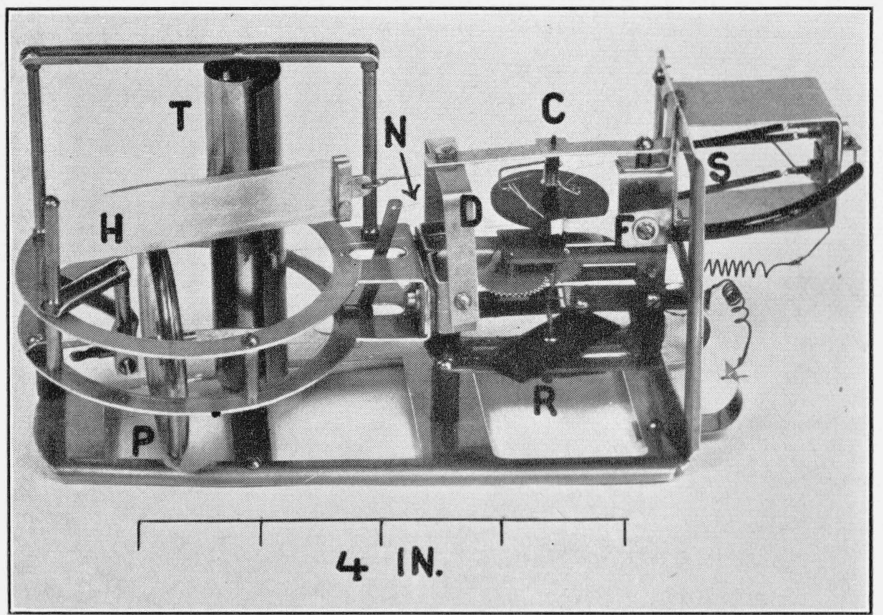

Figure 1.-Olland type radio telemeter driven by electric motor.

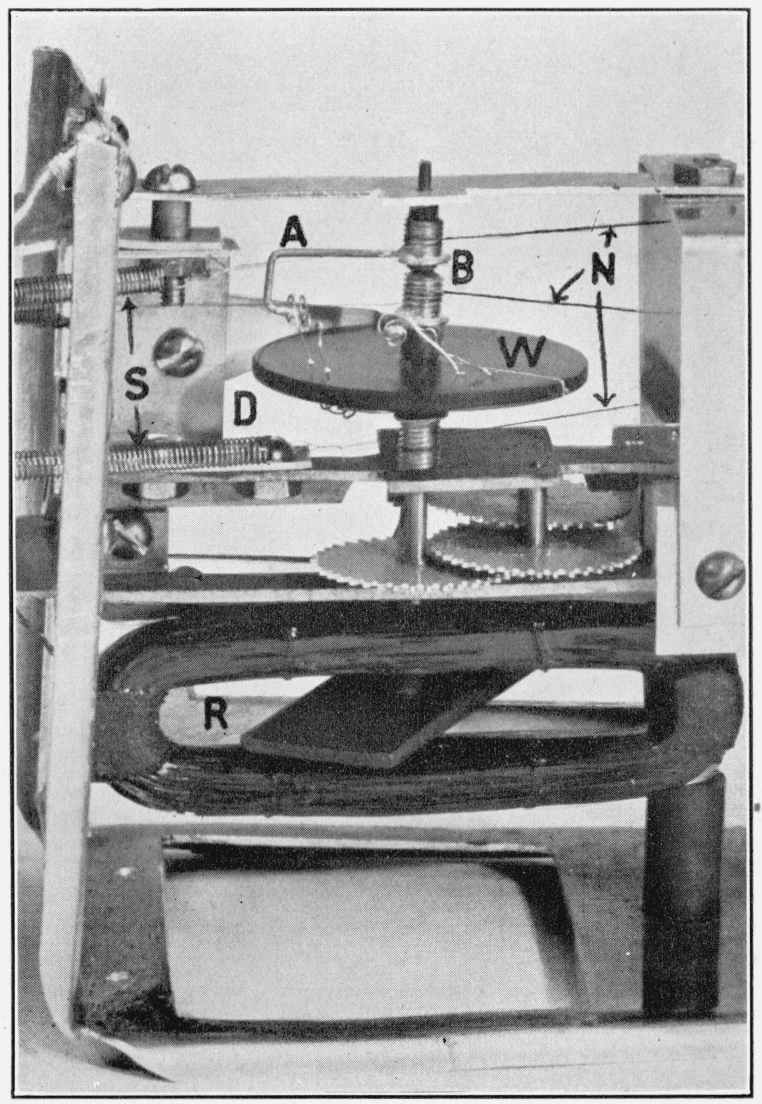

Figure 2.-Detailed view of contact system of Olland type telemeter. 


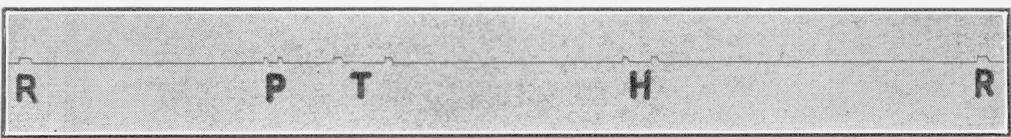

FIGURE 3.- Sample of actual tape record from telemeter, showing one Olland cycle

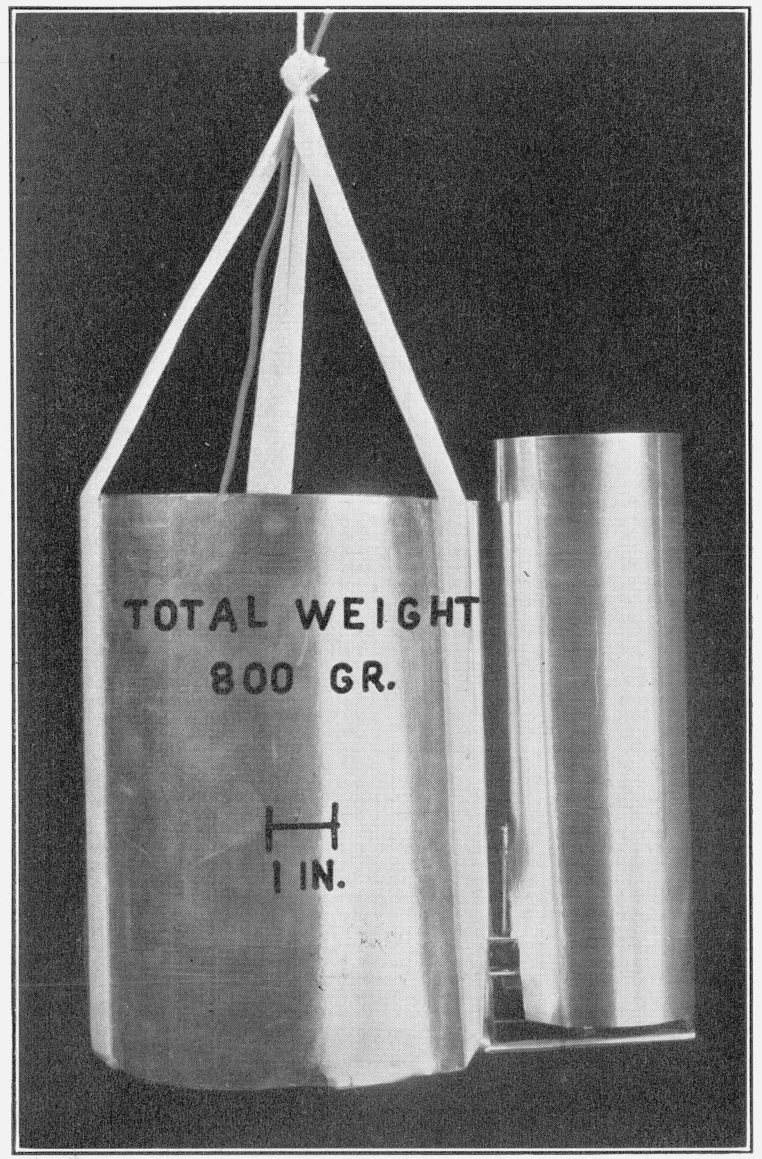

FiguRE 4.-Assembled radio meteorograph ready for release. 
released almost exclusively when the sun is above the horizon. However, the majority of radio-meteorographic observations are made by the Weather Bureau at night, and therefore the corrugated-paper inclosure was adopted to reduce heat loss as much as possible. In this way satisfactory results have been obtained without the use of auxiliary means of heating the interior.

With such an arrangement it becomes necessary to design the telemeter so that the sensitive elements can be outside the thermal inclosure and the motor and contacts inside. Our method for accomplishing this can be explained by reference to figures 1 and 2 , which are two views of the telemeter. The electric motor, of which $R$ is the rotor, is mounted in a light aluminum frame used to support also the pressure, temperature, and humidity elements, $P, T$, and $H$. The contact system, $C$, shown in more detail in figure 2 , is mounted directly on the output spindle of the motor, which turns at a speed of 4 revolutions per minute, giving four complete sets of measurements per minute. The contact system consists of a Bakelite disk, $D$, rigidly attached to the output spindle and turning with it. Imbedded radially in this disk is the platinum wire, $W$, which is in electrical contact at one end with the motor spindle. The exposed side of this wire, which is bent around and similarly imbedded in the lower surface of the disk, $D$, is polished down even with the surface of the disk. This permits electrical contact with the indicating arms without disturbing their angular position. These indicating arms, as shown at $A$, figure 2 , are fastened to brass sleeves, $B$, threaded on the outside and provided with Bakelite bushings inside. Each of these arms is tipped with a double prong of fine platinum-iridium wire. The Bakelite bushings, lubricated with dry graphite, fit smoothly on the output spindle of the motor. The threaded brass sleeves are connected with their respective indicating elements by the fine Nichrome wires, $N$, each one of which passes from the free end of a sensitive element around a separate threaded sleeve, $B$, and is attached to a tension spring, $S$, anchored through an insulator to the right-hand side of the frame in figure 1. In this way linear displacements of the sensitive elements are transformed into angular displacements of the indicating arms of the contact system so that backlash is reduced and the number of moving parts is kept at a minimum. Since the spindle on which the indicating arms are pivoted is in constant rotation, and as there is some vibration in addition from the motor, no difficulty has been encountered in securing a reliably responsive action from the contact arms.

Since the electric motor has considerable power, the limit to the pressure of the tips of the indicating arms on the Bakelite disk is determined by the indicating systems. This pressure must not be so great as to cause dragging of the contact arms. By use of the platinum-iridium tips mentioned above, it has been found easy to obtain reliable electric contacts with moderate pressures. To insure the stability of the indicating systems relatively powerful elements have been used. The bimetallic spiral, T, (fig. 1) and the pressure diaphragm, $P$, have been designed to give strong positive action under all conditions. For the humidity element, $H$, goldbeaters skin has been used, which can be readily cut to a size of sufficient strength and has been found to be several times as responsive as human hair. Its response to changing conditions of humidity has also been found to be 
more definite. The diameter of the threaded boss sleeves, $B$, has been chosen with relation to the linear travel of the sensitive elements to give about $1 \frac{1}{2}$ turns of the indicating arms for the required ranges of temperature and pressure. The humidity arm is limited to $\frac{1}{2}$ turn, which has been found quite satisfactory.

The electrical circuit for keying the transmitter consists of the insulated anchor springs, $S$, and Nichrome wire cables which are in electrical contact with the brass sleeves, $B$, and therefore also with the indicating arms, $A$. The platinum-iridium tips of these arms contact the wire, $W$, which is grounded to the motor frames. Thus the keying leads are attached, one to the remote ends of the anchor springs in parallel and the other to the frame. An additional contact at $F$ is fixed and connected to the insulated ends of the anchor springs. This provides the reference signal to mark off the separate Olland cycles on the record.

The double prong of platinum-iridium mounted at the tip of each indicating arm by means of which electrical contact is made with wire, $W$, has a spacing which is different for each of the three arms. Thus a different double contact is made for the indication for each element which can be identified at once on the record. This is of great value both in calibration and in observations, particularly where, as in the present case, the indicating arms cover more than one Olland cycle. A further convenience in calibration, where each element is calibrated separately, is that the other two may be disconnected so as to give no signal. A typical record for 1 cycle is shown in figure 3 , which shows a strip of actual record. The characteristic spacings of the three doublets, $P, T$, and $H$, are easily observed. Signals, $R R$, are made by the fixed contact marking the beginning and end of an Olland cycle. Stylograph tape is used for recording in a tape chronograph driven by a synchronous motor operated from alternating-current mains.

The framework supporting the sensitive elements is circular so that it may support a cylindrical solar shield of paper covered on both sides with aluminum foil. In the model shown, the distance between the circular frame and the motor spindle has been made as short as convenient. However, this distance may be increased if desired. This might be necessary under some conditions to protect the sensitive elements more effectually from the radiation from the warmer inclosure containing the batteries and transmitter.

The complete radio meteorograph, consisting of telemeter, transmitter, batteries, and antenna, is assembled as shown in figure 4. The small cylinder at the right is the solar shield inclosing the sensitive elements. The other cylindrical container is the thermal inclosure for transmitter motor and batteries. The frame of the telemeter forms a rigid connecting link between the thermal inclosure and the solar shield. The antenna is brought out of the top of the thermal inclosure, permitting it to be stretched straight up along the supporting cord. A top view with cover removed is shown in figure 5, where the sensitive elements $P, T$, and $H$ are labeled. Inside the thermal inclosure may'be seen the oscillator, $O$, of the transmitter and one 45 -volt $B$ battery, $V$. Figure 6 is a bottom view, where the other 45 -volt battery, $V$, and the filament battery, $G$, may be seen.

An advantage of this general arrangement in which the telemeter is treated as a unit is that the telemeter may be used with any trans- 


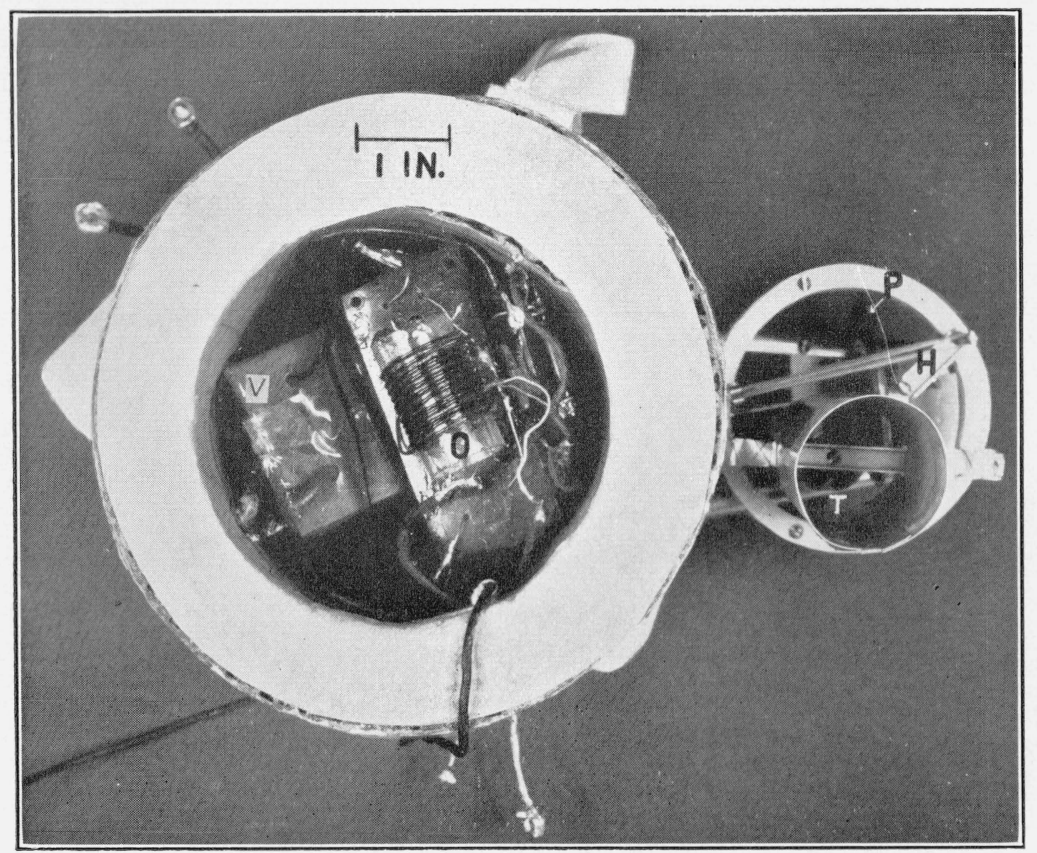

FiguRE 5.-Interior of radio meteorograph viewed from top. Outer solar shield has been removed.

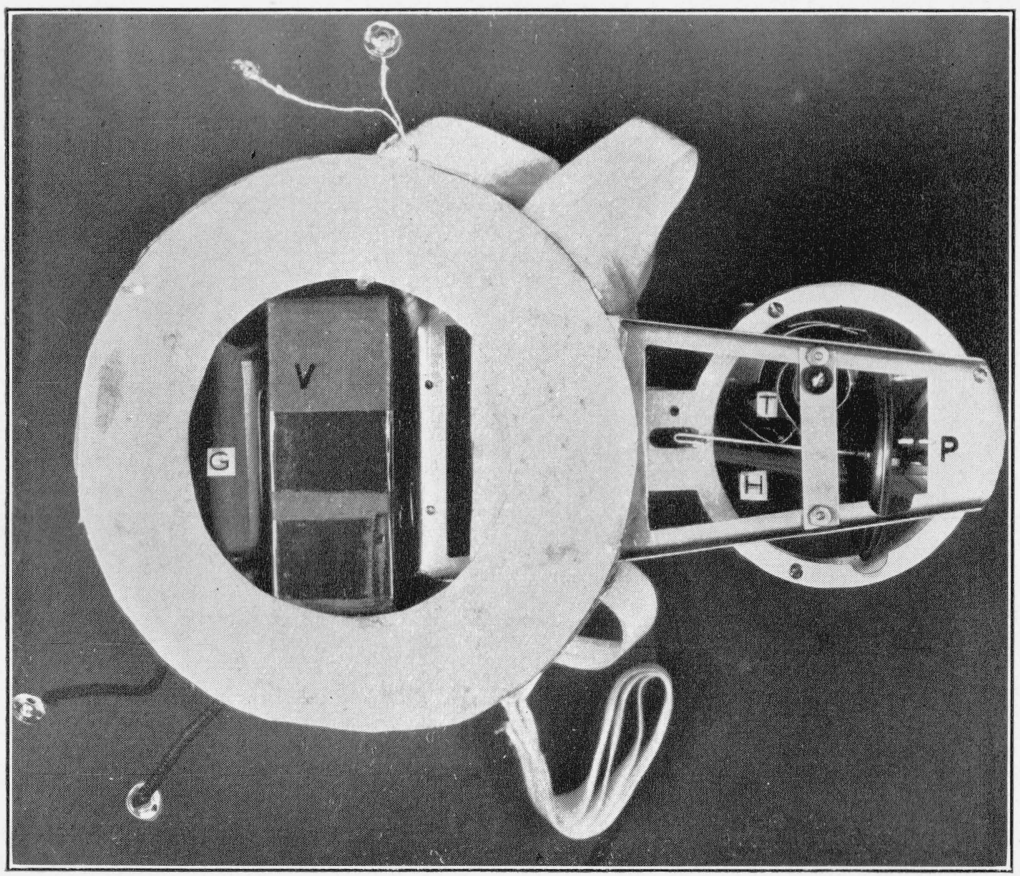

FIGURE 6.-Interior of radio meteorograph viewed from below. 


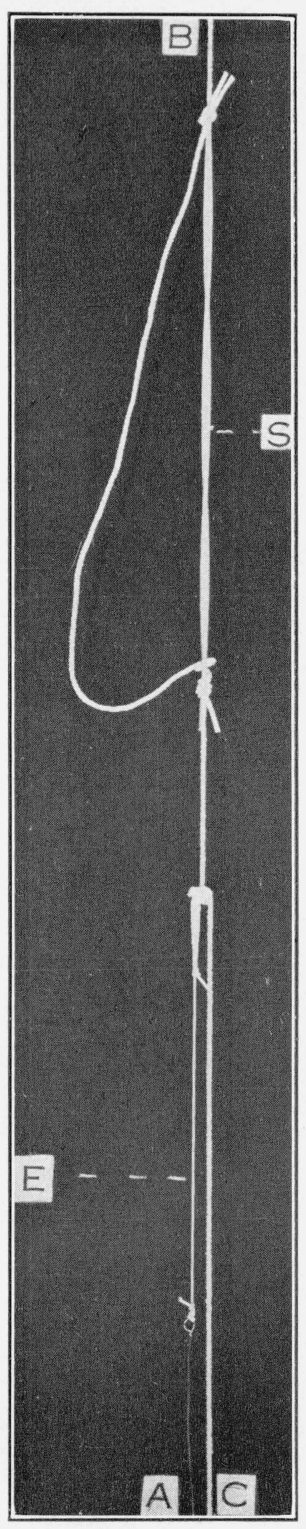

Figure 7.-Arrangement for suspending radio meteorograph from balloon to maintain straight, vertical antenna. 
mitter desired. Furthermore, since they are calibrated independently of the transmitter, a number of calibrated telemeters may be held in reserve ready for use. In those cases where reconditioned instruments are used after recovery from flight, the telemeter may be separated readily from the remainder of the instrument and repaired and recalibrated as a separate unit. Since in the repair of the telemeter and of the transmitter two different types of operations are involved, this is a convenience.

Special attention has been given to the position of the voltage-fed antenna. It has been found desirable to maintain this antenna in a taut vertical position and to prevent excessive swinging of the instruments below in order to preserve strong signals with reasonable stability in frequency. The suspension system shown in figure 7 has been found satisfactory and does not require the antenna to take the full weight of the instrument. $A$ in figure 7 is the antenna and $C$ the load cord, both of which are attached to the instrument some distance below the section shown. $E$ is an elastic cord which holds the antenna stretched and limits the pull upon it. $S$ is a heavy-elastic shock cord by which the whole weight is suspended, which reduces considerably the tendency of the antenna to swing. The cord at $B$ is attached to the balloon or parachute.

The radio meteorograph has been subjected to numerous laboratory tests. These show that the telemeter is capable of measuring temperatures to $\pm 0.5^{\circ} \mathrm{C}$. The response of the humidity element is rapid and exceeds in magnitude by several times that of human hair. The time lag is too great to give very accurate readings with ascensional rates usually used. However, the changes in humidity are very clearly revealed and an accuracy of \pm 5 percent can be obtained under favorable conditions. In a discussion of the precision of the instrument, it should be noted that the demand for accuracy of the pressure element is much greater than that required of the temperature element. It is desirable in many cases to know the pressure to within 1 millibar, if possible, whereas a knowledge of the temperature to the nearest degree is usually adequate. This represents an accuracy of about 0.1 percent of the total pressure range and only 1 percent of the total temperature range. Therefore, in precision tests we have paid most attention to the pressure indications.

The best illustration of the actual precision of which the indicating mechanism used in this radio meteorograph is capable is provided by the authors' experience with a radio barograph constructed in the same way as the radio meteorograph but without temperature or humidity elements. Approximately 50 of these instruments have been sent aloft and about 30 have been returned intact. These returned instruments were recalibrated and the data compared with the original curves. We have found that about 50 percent of the original calibration points reproduce to within \pm 1 millibar and that not more than 10 percent were off by as much as \pm 3 millibars. An actual example of this reproducibility of results is shown in the curve in figure 8 , which shows the original calibration curve of a barograph and the check points determined after the recovery from an ascension to 92,000 feet. None of the check points depart by more than 1 millibar from the original curve. 
To test the effectiveness of the solar shield and thermal insulation of the battery box several test flights have been made with models assembled as described above. Weakened signals of varying frequency due to drop in battery temperatures as well as slowing of the motor would be expected in the absence of sufficient protection for the battery compartment. In all flights strong signals of frequency constant to \pm 60 kilocycles $(0.001 \%)$ were received both on the ascent and also on the descent where the rate of fall was sufficiently slow. In some of the flights the slot cut in the battery box to admit the telemeter was left open. This resulted in a slowing of the motor

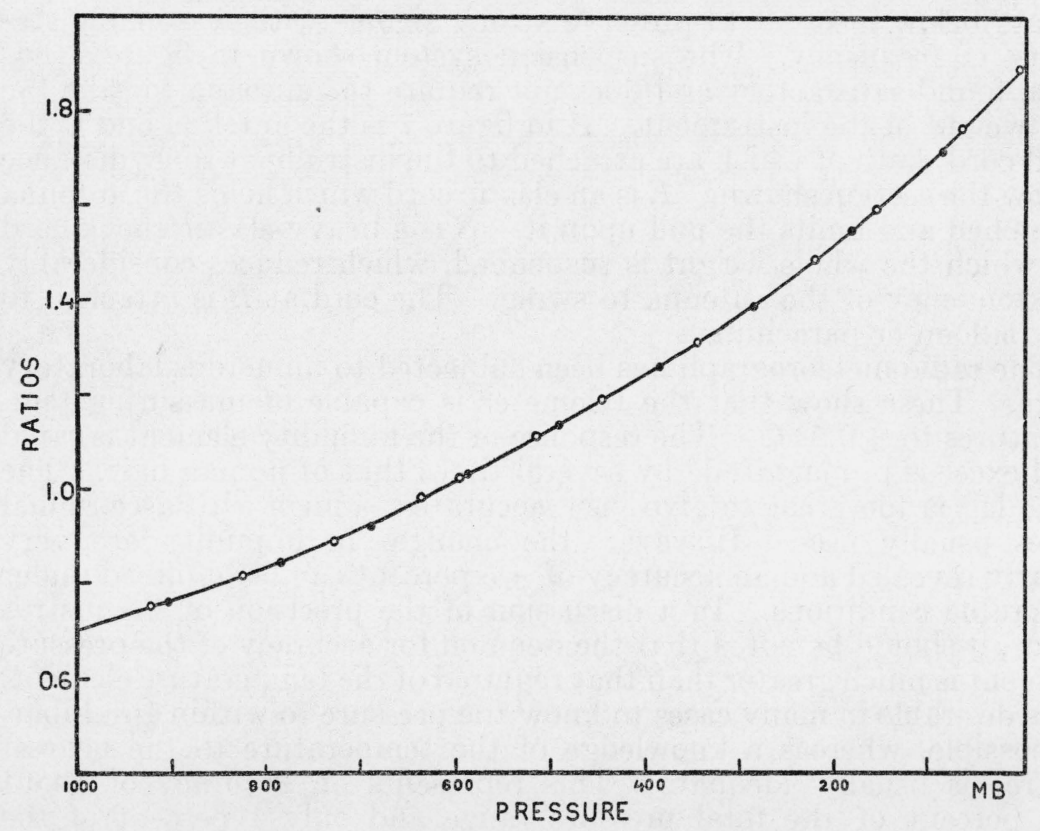

FIGURE 8.-Calibration of curve of pressure element with redetermined points to show accuracy and reproducibility.

Solid circles, original calibration; and open circles, recalibration. Maximum deviation, 1 millibar.

at the higher altitudes. When this opening was properly closed very little change in motor speed was observed.

The question of accuracy under actual working conditions is difficult to deal with in the absence of a standard for comparison. The best we can offer is a comparison of one of our records made on July 20, 1938 at 5:37 a. m. with one made for the Weather Bureau by the Navy, using a Navy type of instrument ${ }^{5}$ released at a point about 8 miles away at 6:00 a. m. This record is shown in figure 9 plotted on an adiabatic chart. There is a consistent difference throughout in the temperatures, ours being above. At the present time we have no adequate explanation for this discrepancy. It is possible that the calibration of one or both instruments used in the above comparisons

B. Diamond, W. S. Hinman, Jr., and F. W. Dunmore, Bul. Am. Meteor. Soc. 18, 73 (1937). 


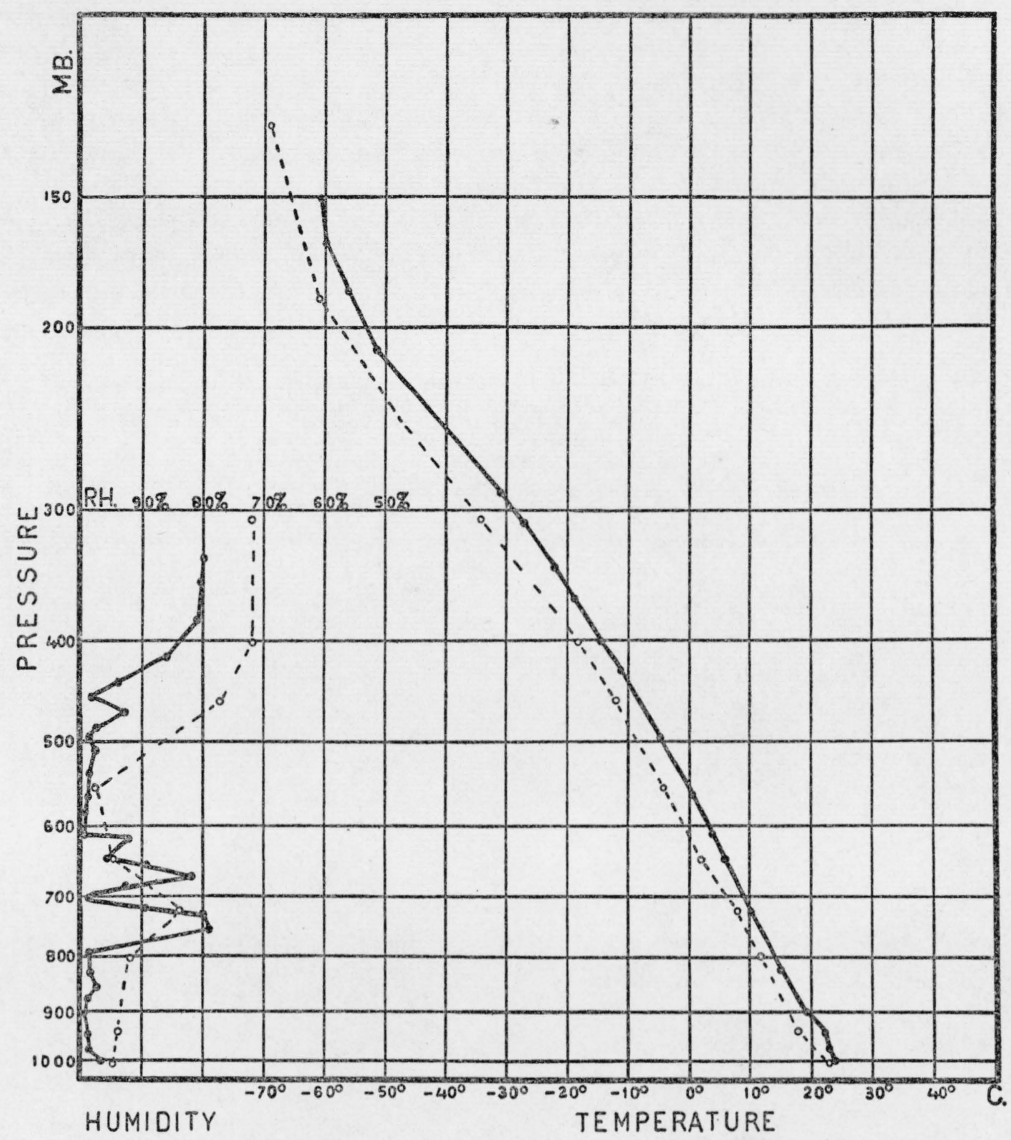

FIGURE 9.-Records of Navy type instrument (dotted lines) plotted on same chart with data (solid lines) obtained by authors' instrument.

Instruments released about the same time at points 8 miles apart.

was in error. The general agreement in trends is very satisfactory and indicates that these methods can be used reliably to obtain upperair data.

We acknowledge the aid of the United States Weather Bureau in providing means for the construction and test of the telemeter and for suggestions regarding improvements and modifications.

Washington, September 28, 1938. 